Pacific

Journal of

Mathematics

EVOLVING CONVEX CURVES TO CONSTANT-WIDTH ONES BY A PERIMETER-PRESERVING FLOW

Laiyuan GaO And Shengliang Pan 


\title{
EVOLVING CONVEX CURVES TO CONSTANT-WIDTH ONES BY A PERIMETER-PRESERVING FLOW
}

\author{
LAIYUAN GAO AND SHENGLIANG PAN
}

\begin{abstract}
This paper deals with a curve evolution problem which, if the curvature of the initial convex curve satisfies a certain pinching condition, keeps the convexity and preserves the perimeter, while increasing the enclosed area of the evolving curve, and which leads to a limiting curve of constant width. In particular, under this flow the limiting curve is a circle if and only if the initial convex curve is centrosymmetric.
\end{abstract}

\section{Introduction}

Denote by $S^{1}$ the unit circle centered at the origin of the Euclidean plane $\mathbb{R}^{2}$. Let $X_{0}(\varphi), \varphi \in S^{1}$, be a closed $C^{\infty}$ curve in the plane. A curve evolution problem is usually defined as

$$
\begin{cases}(\partial X / \partial t)(\varphi, t)=\beta(\varphi, t) N(\varphi, t), & (\varphi, t) \in S^{1} \times(0, T), \\ X(\varphi, 0)=X_{0}(\varphi), & \varphi \in S^{1},\end{cases}
$$

where $X(\varphi, t)=(x(\varphi, t), y(\varphi, t))$ is the position vector of the evolving curve, $N(\varphi, t)$ its unit normal vector field and $\beta(\varphi, t)$ some geometric quantity depending on the evolving curve. Such problems arise in many fields, such as image processing [Cao 2003], phase transitions [Gurtin 1993], etc. In fact, the above evolution problem has been studied extensively, for example, for the popular curve-shortening flow [Gage 1984; Gage and Hamilton 1986; Grayson 1987], the area-preserving flows [Gage 1986; Mao et al. 2013; Ma and Cheng 2014], the perimeter-preserving flows [Pan and Yang 2008; Ma and Zhu 2012] and in other related research [Angenent 1991; Chow and Tsai 1996; Andrews 1998; Urbas 1999; Chao et al. 2013]. One can find more background material in the book [Chou and Zhu 2001].

Let $\theta$ be the tangential angle, i.e., the oriented angle from the positive $x$-axis to the unit tangential vector of the curve. If the initial curve $X_{0}$ is strictly convex then it can be parameterized by $\theta$. In this paper, we will focus on the following curve

This work is supported by the National Science Foundation of China (grant 11171254).

MSC2010: 35K15, 35K55, 53A04.

Keywords: convex curves, curves of constant width, perimeter-preserving curve flow. 
evolution problem in the plane $\mathbb{R}^{2}$ :

$$
\begin{cases}(\partial X / \partial t)(\theta, t)=(w(\theta, t)-\eta(\theta, t)) N(\theta, t), & (\theta, t) \in S^{1} \times(0, T), \\ X(\theta, 0)=X_{0}(\theta), & \theta \in S^{1},\end{cases}
$$

where $w(\theta, t)$ is the width of the evolving curve and $\eta(\theta, t)$ is $\rho(\theta, t)+\rho(\theta+\pi, t)$; here $\rho(\theta, t)$ is the radius of curvature of the curve. Using the Minkowski support function of a convex curve (see [Hsiung 1981; Schneider 1993; Groemer 1996]), one can easily see that $w(\theta, t)-\eta(\theta, t)=-\left(\partial^{2} w / \partial \theta^{2}\right)(\theta, t)$, and thus it is obvious that constant-width curves are invariant (i.e., stable) under this flow.

The following theorem is the main result of our paper.

Main Theorem. Let $X_{0}(\theta)$ be a strictly convex smooth curve in the plane $\mathbb{R}^{2}$ which evolves according to the flow (1-1). Denote by $\rho_{0}(\theta)$ the radius of curvature of $X_{0}(\theta)$ and set

$$
M=\max \left\{\rho_{0}(\theta) \mid \theta \in S^{1}\right\}, \quad m=\min \left\{\rho_{0}(\theta) \mid \theta \in S^{1}\right\} .
$$

If the pinching condition

$$
M<3 m
$$

holds for $X_{0}(\theta)$, i.e., $\frac{1}{3}<m / M \leq 1$, then (1-1) has a global solution $X(\theta, t)$ for $(\theta, t) \in S^{1} \times[0, \infty)$. As time passes, the flow keeps the convexity, preserves the perimeter while increasing the enclosed area of the evolving curve, and makes the curve more and more circular. As the time $t$ goes to infinity, the curve $X(\cdot, t)$ evolves smoothly to a curve of constant width $L_{0} / \pi$, where $L_{0}$ is the perimeter of the initial convex curve $X_{0}(\theta)$. In particular, the limiting curve is a circle if and only if the initial curve is centrosymmetric.

If a smooth simple closed curve evolves under the curve shortening flow then it converges to a round point (see [Gage 1984; Gage and Hamilton 1986; Grayson 1987]). In the cases of nonlocal flows for convex curves, the limiting curves are finite circles (see [Gage 1986; Jiang and Pan 2008; Pan and Zhang 2010; Ma and Cheng 2014]). Forming a striking contrast to these researches, although in the present case the evolving curve keeps its convexity and becomes more and more circular, the limiting curve of the flow is only of constant width rather than being a circle.

This paper is organized as follows. In Section 2, we will compute the evolution equations of the commonly used geometric quantities, and reduce the nonlinear problem (1-1) to the Cauchy problem

$$
\left\{\begin{array}{l}
(\partial \rho / \partial t)(\theta, t)=\left(\partial^{2} \eta / \partial \theta^{2}\right)(\theta, t), \\
(\partial \eta / \partial t)(\theta, t)=2\left(\partial^{2} \eta / \partial \theta^{2}\right)(\theta, t), \\
\rho(\theta, 0)=\rho_{0}(\theta), \\
\eta(\theta, 0)=\eta_{0}(\theta),
\end{array}\right.
$$


where $\theta$ is the tangential angle and $(\theta, t)$ is in $S^{1} \times[0, T)$. In Section 3 , we will show that the Cauchy problem (1-3) has a bounded positive solution in $S^{1} \times[0,+\infty)$, provided that condition (1-2) holds. We will prove that the evolving curve maintains its convexity and is of the same length as the initial convex curve. As time tends to infinity, the asymptotic behavior of the evolving curve will be considered. In Section 4 we will give several examples.

\section{Some preparations}

In this section, we will first calculate the evolution equations of the commonly used geometric quantities, and then give the equivalence between the curve evolution problem (1-1) and the Cauchy problem (1-3). Now, we suppose that there exists a family of convex curves $X(\varphi, t)$ evolving according to (1-1).

To make the tangential angle $\theta$ a variable independent of time $t$, let us consider the following flow instead of (1-1):

$$
\left\{\begin{array}{l}
(\partial \tilde{X} / \partial t)(\theta, t)=\alpha(\theta, t) T(\theta, t)+(w(\theta, t)-\eta(\theta, t)) N(\theta, t) \\
\tilde{X}(\theta, 0)=X_{0}(\theta)
\end{array}\right.
$$

where $\alpha=\alpha(\theta, t)$ is to be determined. Set $\beta(\theta, t)=w(\theta, t)-\eta(\theta, t)$. By [Chou and Zhu 2001, Proposition 1.1, p. 6], the solution of $(2-1), \tilde{X}(\cdot, t)$, differs from the solution of $(1-1), X(\cdot, t)$, only by altering the parametrization. Therefore, we just need to calculate the evolution equations of $\rho$ and $\eta$ under the flow (2-1).

Let $s$ be the arc length of the curve $\tilde{X}(\cdot, t)$. The metric of the curve is given by $g(\varphi, t)=\|\partial \tilde{X} / \partial \varphi\|$. From the Frenet formulae it follows that

$$
\begin{aligned}
\frac{\partial g}{\partial t} & =\frac{1}{g}\left\langle\frac{\partial}{\partial t} \frac{\partial \tilde{X}}{\partial \varphi}, \frac{\partial \tilde{X}}{\partial \varphi}\right\rangle=\frac{1}{g}\left\langle\frac{\partial}{\partial \varphi}(\alpha T+\beta N), \frac{\partial \tilde{X}}{\partial \varphi}\right\rangle \\
& =\frac{1}{g}\left\langle g \frac{\partial}{\partial s}(\alpha T+\beta N), g T\right\rangle=\left(\frac{\partial \alpha}{\partial s}-\beta \kappa\right) g .
\end{aligned}
$$

Therefore, one gets

$$
\begin{aligned}
\frac{\partial}{\partial t} \frac{\partial}{\partial s} & =\frac{\partial}{\partial t}\left(\frac{1}{g} \frac{\partial}{\partial \varphi}\right)=-\frac{1}{g^{2}} \frac{\partial g}{\partial t} \frac{\partial}{\partial \varphi}+\frac{1}{g} \frac{\partial}{\partial t} \frac{\partial}{\partial \varphi} \\
& =-\frac{1}{g} \frac{\partial g}{\partial t} \frac{\partial}{\partial s}+\frac{1}{g} \frac{\partial}{\partial \varphi} \frac{\partial}{\partial t}=\left(-\frac{\partial \alpha}{\partial s}+\beta \kappa\right) \frac{\partial}{\partial s}+\frac{\partial}{\partial s} \frac{\partial}{\partial t}
\end{aligned}
$$

and

$$
\begin{aligned}
\frac{\partial T}{\partial t} & =\frac{\partial}{\partial t} \frac{\partial X}{\partial s}=\frac{\partial}{\partial s} \frac{\partial X}{\partial t}+\left(-\frac{\partial \alpha}{\partial s}+\beta \kappa\right) \frac{\partial X}{\partial s} \\
& =\frac{\partial}{\partial s}(\alpha T+\beta N)+\left(-\frac{\partial \alpha}{\partial s}+\beta \kappa\right) T=\left(\alpha \kappa+\frac{\partial \beta}{\partial s}\right) N
\end{aligned}
$$




$$
\frac{\partial N}{\partial t}=\left\langle\frac{\partial N}{\partial t}, T\right\rangle T+\left\langle\frac{\partial N}{\partial t}, N\right\rangle N=-\left\langle N, \frac{\partial T}{\partial t}\right\rangle T+0=-\left(\alpha \kappa+\frac{\partial \beta}{\partial s}\right) T .
$$

Since the Frenet frame can be expressed via the tangential $\theta$ as $T=(\cos \theta, \sin \theta)$, $N=(-\sin \theta, \cos \theta)$, one can obtain the Frenet formulae

$$
\frac{\partial T}{\partial \theta}=N, \quad \frac{\partial N}{\partial \theta}=-T .
$$

The definition of curvature $\kappa$ implies that $\partial \theta / \partial s=\kappa$ or $\partial s / \partial \theta=\rho$. Noticing that

$$
\left(\alpha \kappa+\frac{\partial \beta}{\partial s}\right) N=\frac{\partial T}{\partial t}=\frac{\partial}{\partial t}(\cos \theta, \sin \theta)=(-\sin \theta, \cos \theta) \frac{\partial \theta}{\partial t}=\frac{\partial \theta}{\partial t} N,
$$

one obtains that

$$
\frac{\partial \theta}{\partial t}=\alpha \kappa+\frac{\partial \beta}{\partial s} .
$$

From (2-3), if we set $\alpha=-(1 / \kappa)(\partial \beta / \partial s)=-\partial \beta / \partial \theta$, then the tangential angle $\theta$ is independent of $t$ and so are $T$ and $N$. The evolution equation of the Minkowski support function $p$ of the evolving curve is given by

$$
\frac{\partial p}{\partial t}=-\frac{\partial}{\partial t}\langle X, N\rangle=-\left\langle\frac{\partial X}{\partial t}, N\right\rangle+0=-\beta=\eta-w .
$$

Since

$$
\begin{aligned}
\frac{\partial p}{\partial \theta} & =-\left\langle\frac{\partial X}{\partial \theta}, N\right\rangle-\left\langle X, \frac{\partial N}{\partial \theta}\right\rangle=\langle X, T\rangle, \\
\frac{\partial^{2} p}{\partial \theta^{2}} & =\frac{\partial}{\partial \theta}\langle X, T\rangle=\left\langle\frac{\partial X}{\partial s} \frac{\partial s}{\partial \theta}, T\right\rangle+\langle X, N\rangle=\rho-p,
\end{aligned}
$$

one gets

$$
\rho=\frac{\partial^{2} p}{\partial \theta^{2}}+p
$$

and

$$
\begin{aligned}
\eta(\theta, t) & =\rho(\theta, t)+\rho(\theta+\pi, t) \\
& =p(\theta, t)+\frac{\partial^{2} p}{\partial \theta^{2}}(\theta, t)+p(\theta+\pi, t)+\frac{\partial^{2} p}{\partial \theta^{2}}(\theta+\pi, t) \\
& =\frac{\partial^{2} w}{\partial \theta^{2}}(\theta, t)+w(\theta, t) .
\end{aligned}
$$

From the evolution equation of the support function $p$ and from the definition of width, $w(\theta, t):=p(\theta, t)+p(\theta+\pi, t)$, we have

$$
\frac{\partial w}{\partial t}=2 \eta-2 w=2 \frac{\partial^{2} w}{\partial \theta^{2}}
$$


and

$$
\begin{aligned}
\frac{\partial \rho}{\partial t} & =\frac{\partial}{\partial t}\left(\frac{\partial^{2} p}{\partial \theta^{2}}+p\right)=\frac{\partial^{2}}{\partial \theta^{2}} \frac{\partial p}{\partial t}+\frac{\partial p}{\partial t}=\frac{\partial^{2}}{\partial \theta^{2}}(\eta-w)+(\eta-w) \\
& =\frac{\partial^{2} \eta}{\partial \theta^{2}}-\frac{\partial^{2} w}{\partial \theta^{2}}+\eta-w=\frac{\partial^{2} \eta}{\partial \theta^{2}} \\
\frac{\partial \eta}{\partial t} & =\frac{\partial}{\partial t}(\rho(\theta, t)+\rho(\theta+\pi, t))=2 \frac{\partial^{2} \eta}{\partial \theta^{2}} .
\end{aligned}
$$

Now, we can conclude that if there is a family of convex curves $X(\cdot, t)$ evolving according to the flow (2-1), then the Cauchy problem (1-3) is solvable for some $T>0$. The following theorem can tell us that the contrary also holds.

Theorem 2.1. The curve evolution problem (1-1) is equivalent to the Cauchy problem (1-3) for some $T>0$, if the initial curve $X(\varphi, 0)=X_{0}(\varphi)$ is smooth and strictly convex.

Proof. We just need to prove that if the Cauchy problem (1-3) has a solution $\eta(\theta, t)$ for some $T>0$ then the evolution problem (2-1) is solvable for $(\theta, t) \in S^{1} \times[0, T)$. Define a family of curves $\tilde{X}(\theta, t)=(\tilde{x}(\theta, t), \tilde{y}(\theta, t))+\left(C_{1}(t), C_{2}(t)\right)$ by setting

$$
\begin{array}{rlrl}
\tilde{x}(\theta, t) & =\int_{0}^{\theta} \rho(\phi, t) \cos \phi d \phi, & \tilde{y}(\theta, t) & =\int_{0}^{\theta} \rho(\phi, t) \sin \phi d \phi . \\
C_{1}(t) & =\int_{0}^{t}\left(\frac{\partial \eta}{\partial \theta}(0, \tau)-\frac{\partial w}{\partial \theta}(0, \tau)\right) d \tau, & C_{2}(t)=\int_{0}^{t}(w(0, \tau)-\eta(0, \tau)) d \tau .
\end{array}
$$

Direct computation gives us

$$
\begin{aligned}
\frac{\partial \tilde{x}}{\partial t} & =\int_{0}^{\theta} \frac{\partial \rho}{\partial t}(\phi, t) \cos \phi d \phi=\int_{0}^{\theta} \frac{\partial^{2} \eta}{\partial \phi^{2}}(\phi, t) \cos \phi d \phi \\
& =\frac{\partial \eta}{\partial \theta} \cos \theta-\frac{\partial \eta}{\partial \theta}(0, t)+\eta \sin \theta-\int_{0}^{\theta} \eta \cos \phi d \phi \\
& =\frac{\partial \eta}{\partial \theta} \cos \theta-\frac{\partial \eta}{\partial \theta}(0, t)+\eta \sin \theta-\int_{0}^{\theta}\left(\frac{\partial^{2} w}{\partial \phi^{2}}+w\right) \cos \phi d \phi \\
& =\left(\frac{\partial \eta}{\partial \theta}-\frac{\partial w}{\partial \theta}\right) \cos \theta-\left(\frac{\partial \eta}{\partial \theta}(0, t)-\frac{\partial w}{\partial \theta}(0, t)\right)+(\eta-w) \sin \theta
\end{aligned}
$$

And similarly, one can get

$$
\frac{\partial \tilde{y}}{\partial t}=\left(\frac{\partial \eta}{\partial \theta}-\frac{\partial w}{\partial \theta}\right) \sin \theta+(\eta(0, t)-w(0, t))+(w-\eta) \cos \theta
$$


So the curve $\tilde{X}(\theta, t)$ satisfies

$$
\frac{\partial \tilde{X}}{\partial t}=-\frac{\partial}{\partial \theta}(w-\eta) T+(w-\eta) N,
$$

where $\{T, N\}$ is the Frenet frame of the curve $\tilde{X}$, which implies that the flow (2-1) has a solution since we have chosen $\alpha=-(1 / \kappa)(\partial \beta / \partial s)=-\partial \beta / \partial \theta$. Therefore the original problem (1-2) is also solvable on $S^{1} \times[0, T)$.

\section{Global existence and convergence of the flow}

Because we can reduce the curve evolution problem (1-1) to a Cauchy problem (1-3) for small $t$, the local existence of the flow (1-1) is a direct corollary of the classical theory of heat equations. In this section, we will first prove that problem (1-1) has a unique, convex and smooth solution curve $X(\varphi, t)$ on $S^{1} \times[0,+\infty)$; i.e., the Cauchy problem (1-3) has a positive and smooth solution $(\rho(\cdot, t), \eta(\cdot, t))$ for $t \geq 0$, provided that the pinching condition (1-2) holds for the initial curve. Then we will show that the curve $X(\cdot, t)$ evolves to a constant-width curve smoothly.

Lemma 3.1. The Cauchy problem (1-3) has a global solution $\rho(\cdot, t)$ for $t \geq 0$. If the pinching condition (1-2) holds, then there exist two positive constants $C_{1}$ and $C_{2}$ such that

$$
C_{1} \leq \rho(\theta, t) \leq C_{2}
$$

for $(\theta, t) \in S^{1} \times[0,+\infty)$.

Proof. The local and global existence of solutions for the Cauchy problem (1-3) is a direct corollary of the classical theory for heat equations. Suppose (1-3) has a positive solution $\rho(\theta, t)$ on $S^{1} \times[0, T)$ for some $T>0$. Since $\partial \eta / \partial t=$ $2 \partial^{2} \eta / \partial \theta^{2}$ and $\eta(\theta, 0)=\eta_{0}(\theta)$ is a positive smooth function, we know that $\eta(\theta, t)$ is defined on $S^{1} \times[0,+\infty)$ and is smooth. Furthermore, by the maximum principle, $n \leq \eta(\theta, t) \leq N$, where $n:=\min \left\{\eta_{0}(\theta) \mid \theta \in S^{1}\right\}, N:=\max \left\{\eta_{0}(\theta) \mid \theta \in S^{1}\right\}$. By the evolution equation of $\eta$ and Writinger's inequality, we have

$$
\frac{d}{d t} \int_{0}^{2 \pi}\left(\frac{\partial^{k} \eta}{\partial \theta^{k}}\right)^{2} d \theta=-4 \int_{0}^{2 \pi}\left(\frac{\partial^{k+1} \eta}{\partial \theta^{k+1}}\right)^{2} d \theta \leq-16 \int_{0}^{2 \pi}\left(\frac{\partial^{k} \eta}{\partial \theta^{k}}\right)^{2} d \theta,
$$

where $k$ is a positive integer. And thus

$$
\int_{0}^{2 \pi}\left(\frac{\partial^{k} \eta}{\partial \theta^{k}}\right)^{2} d \theta \leq\left[\int_{0}^{2 \pi}\left(\frac{\partial^{k} \eta_{0}}{\partial \theta^{k}}\right)^{2} d \theta\right] e^{-16 t}
$$

Hence, by Sobolev's inequality, one gets

$$
\max _{\theta \in[0,2 \pi]}\left|\frac{\partial^{k} \eta}{\partial \theta^{k}}\right| \leq C(k) e^{-8 t}
$$


where

$$
C(k)=\frac{1}{\sqrt{2 \pi}}\left(\int_{0}^{2 \pi}\left(\frac{\partial^{k} \eta_{0}}{\partial \theta^{k}}\right)^{2} d \theta\right)^{\frac{1}{2}}+\sqrt{2 \pi}\left(\int_{0}^{2 \pi}\left(\frac{\partial^{k+1} \eta_{0}}{\partial \theta^{k+1}}\right)^{2} d \theta\right)^{\frac{1}{2}} .
$$

Notice that $\partial \rho / \partial t=\partial^{2} \eta / \partial \theta^{2} \leq C(2) e^{-8 t}$; i.e.,

$$
\rho(\theta, t) \leq \rho_{0}(\theta)+C(2) \frac{1}{8}\left(1-e^{-8 t}\right) \leq M+\frac{1}{8} C(2),
$$

where $M=\max \left\{\rho_{0}(\theta) \mid \theta \in S^{1}\right\}$. Letting $C_{2}=M+\frac{1}{8} C(2)$ gives us

$$
\rho(\theta, t) \leq C_{2} \text {. }
$$

From (1-3), we get $\partial \rho / \partial t=\partial^{2} \eta / \partial \theta^{2}=\frac{1}{2} \partial \eta / \partial t$, which yields

$$
\rho=\rho_{0}+\frac{1}{2}\left(\eta-\eta_{0}\right) \text {. }
$$

By the maximum principle, we know that $\eta(\theta, t) \geq \min \left\{\eta_{0}(\theta) \mid \theta \in S^{1}\right\}>0$. If $\eta_{0}(\theta)$ attains its minimum $n$ at $\theta_{n}$, then one has

$$
\begin{aligned}
\rho(\theta, t) & =\rho_{0}(\theta)+\frac{1}{2}\left(\eta(\theta, t)-\eta_{0}(\theta)\right) \\
& \geq \rho_{0}(\theta)+\frac{1}{2}\left(n-\rho_{0}(\theta)-\rho_{0}(\theta+\pi)\right) \\
& =\frac{1}{2}\left(\rho_{0}(\theta)+\rho_{0}\left(\theta_{n}\right)+\rho_{0}\left(\theta_{n}+\pi\right)-\rho_{0}(\theta+\pi)\right) \\
& \geq \frac{1}{2}(3 m-M)>0 .
\end{aligned}
$$

Namely, there exists a positive constant $C_{1}=\frac{1}{2}(3 m-M)$ such that

$$
\rho(\theta, t) \geq C_{1} .
$$

Combining (3-3) and (3-5), we complete the proof of (3-1).

Corollary 3.2. If the pinching condition (1-2) holds for the strictly convex initial curve $X_{0}(\varphi)$, then the problem (1-1) has a unique global solution $X(\varphi, t)$ on $S^{1} \times[0,+\infty)$ and $X(\cdot, t)$ is a strictly convex curve for all $t>0$.

Lemma 3.3. Under the condition of Corollary 3.2, the convex evolving curve converges to a constant-width curve smoothly.

Proof. By the evolution equation of $w$ and the closing condition of the evolving curve, we have almost the same estimate for $w$ as that for $\eta$ :

$$
\max _{\theta \in[0,2 \pi]}\left|\frac{\partial^{k} w}{\partial \theta^{k}}\right| \leq C\left(X_{0}, k\right) e^{-8 t},
$$

where $C\left(X_{0}, k\right)$ is a positive constant depending only on the initial data $X_{0}$ and $k$. By the Arzelà-Ascoli theorem, there exists a subsequence $\left\{w\left(\theta, t_{i}\right)\right\}$ convergent as $t_{i}$ goes to infinity. Since $\lim _{t \rightarrow \infty}|\partial w / \partial \theta|=0, \lim _{t_{i} \rightarrow \infty} w\left(\theta, t_{i}\right)$ equals some constant. 
Noticing that $\int_{0}^{2 \pi} w(\theta, t) d \theta=2 L_{0}$, we obtain that $\lim _{t_{i} \rightarrow \infty} w\left(\theta, t_{i}\right)=L_{0} / \pi$. Since this equality holds for any convergent subsequence of $\{w(\theta, t)\}$, we can claim that $\{w(\theta, t)\}$ is convergent:

$$
\lim _{t \rightarrow \infty} w(\theta, t)=\frac{L_{0}}{\pi}
$$

Similarly, we also have

$$
\lim _{t \rightarrow \infty} \eta(\theta, t)=\frac{L_{0}}{\pi}
$$

From (3-4) it follows that

$$
\lim _{t \rightarrow \infty} \rho(\theta, t)=\frac{L_{0}}{2 \pi}+\frac{1}{2}\left(\rho_{0}(\theta)-\rho_{0}(\theta+\pi)\right) .
$$

Since $M<3 m$ (condition (1-2)), one gets

$$
\lim _{t \rightarrow \infty} \rho(\theta, t) \geq m+\frac{1}{2}(m-M)>0 .
$$

By (3-6) and (3-10), the limit of the evolving curve is convex and is of constant width. By (3-4) and (3-9), we have

$$
\rho(\theta, t)-\lim _{t \rightarrow \infty} \rho(\theta, t)=\frac{1}{2}\left(\eta(\theta, t)-\frac{L_{0}}{\pi}\right) .
$$

Thus that the evolving curve converges smoothly is a corollary of (3-2).

Lemma 3.4. Under the condition of Corollary 3.2, the flow (1-1) keeps the perimeter of the evolving curve $X$ and increases the enclosed area.

Proof. Let $L(t)$ be the perimeter of the evolving curve $X(\cdot, t)$ and $A(t)$ the enclosed area. The variational formulae of $L(t)$ and $A(t)$ in [Gage 1986] give us

$$
\frac{d L}{d t}=-\int_{0}^{L} \beta \kappa d s, \quad \frac{d A}{d t}=-\int_{0}^{L} \beta d s .
$$

Under the flow (1-1), the perimeter evolves according to

$$
\frac{d L}{d t}=-\int_{0}^{2 \pi}(w(\theta, t)-\eta(\theta, t)) d \theta=\int_{0}^{2 \pi} \frac{\partial^{2} w}{\partial \theta^{2}}(\theta, t) d \theta=0,
$$


which implies that the flow (1-1) keeps the perimeter of the evolving curve. By Gage's variational formulae (3-11), the enclosed area evolves according to

$$
\begin{aligned}
\frac{d A}{d t} & =-\int_{0}^{2 \pi}(w(\theta, t)-\eta(\theta, t)) \rho(\theta, t) d \theta=\int_{0}^{2 \pi} \frac{\partial^{2} w}{\partial \theta^{2}}(\theta, t) \rho(\theta, t) d \theta \\
& =\int_{0}^{\pi} \frac{\partial^{2} w}{\partial \theta^{2}}(\theta, t) \rho(\theta, t) d \theta+\int_{\pi}^{2 \pi} \frac{\partial^{2} w}{\partial \theta^{2}}(\theta, t) \rho(\theta, t) d \theta \\
& =\int_{0}^{\pi} \frac{\partial^{2} w}{\partial \theta^{2}}(\theta, t) \rho(\theta, t) d \theta+\int_{0}^{\pi} \frac{\partial^{2} w}{\partial \xi^{2}}(\xi+\pi, t) \rho(\xi+\pi, t) d \xi \\
& =\int_{0}^{\pi} \frac{\partial^{2} w}{\partial \theta^{2}}(\theta, t)(\rho(\theta, t)+\rho(\theta+\pi, t)) d \theta \\
& =\int_{0}^{\pi} \frac{\partial^{2} w}{\partial \theta^{2}}(\theta, t)\left(\frac{\partial^{2} w}{\partial \theta^{2}}(\theta, t)+w(\theta, t)\right) d \theta \\
& =\int_{0}^{\pi}\left(\frac{\partial^{2} w}{\partial \theta^{2}}(\theta, t)\right)^{2} d \theta-\int_{0}^{\pi}\left(\frac{\partial w}{\partial \theta}(\theta, t)\right)^{2} d \theta,
\end{aligned}
$$

where the fact that $w(\theta, t)$ is a periodic function with period $\pi$ with respect to $\theta$ is used. Now, the Wirtinger inequality implies $d A / d t \geq 0$. Namely, flow (1-1) increases the area enclosed by the evolving curve.

From the previous lemma, it follows that

$$
\frac{d}{d t}\left(L^{2}-4 \pi A\right) \leq 0,
$$

which tells us that the isoperimetric deficit of the evolving curve is decreasing and thus the curve becomes more and more circular during the evolution process.

Generally speaking, the pinching condition (1-2) can not be omitted, because we have a lot of convex curves such that the right-hand side of (3-9) is negative for some $\theta$. However, an example in the next section shows that the pinching inequality (1-2) is just a sufficient condition to guarantee the global existence of convex curve $X(\cdot, t)$. We do not know how to weaken this condition.

Next, we will follow the idea from [Lin and Tsai 2009] to study the geometric behavior of the flow (1-1) (using Fourier series). Now suppose that (1-1) has a global solution on $S^{1} \times[0,+\infty)$ and that each evolving curve is strictly convex.

The Fourier expansion of the support function $p(\theta, t)$ of the evolving curve can be written as

$$
p(\theta, t)=\frac{L_{0}}{2 \pi}+\sum_{k=1}^{\infty}\left[a_{k}(t) \cos (k \theta)+b_{k}(t) \sin (k \theta)\right],
$$


where $\theta$ is the tangential angle. By the definitions of width and radius of curvature, we have

$$
\begin{aligned}
& \rho(\theta, t)=\frac{\partial^{2} p}{\partial \theta^{2}}(\theta, t)+p(\theta, t)=\frac{L_{0}}{2 \pi}+\sum_{k=1}^{\infty}\left[a_{k}(t) \cos (k \theta)+b_{k}(t) \sin (k \theta)\right]\left(1-k^{2}\right), \\
& w(\theta, t)=p(\theta, t)+p(\theta+\pi, t)=\frac{L_{0}}{\pi}+2 \sum_{k=1}^{\infty}\left[a_{2 k}(t) \cos (2 k \theta)+b_{2 k}(t) \sin (2 k \theta)\right] .
\end{aligned}
$$

Since $\partial w / \partial t=2\left(\partial^{2} w / \partial \theta^{2}\right)$, we have, by comparing the coefficients of both sides,

$$
w(\theta, t)=\frac{L_{0}}{\pi}+2 \sum_{k=1}^{\infty}\left[a_{2 k}(0) \cos (2 k \theta)+b_{2 k}(0) \sin (2 k \theta)\right] e^{-8 k^{2} t} .
$$

Therefore,

$$
\begin{aligned}
\eta(\theta, t) & =\frac{\partial^{2} w}{\partial \theta^{2}}(\theta, t)+w(\theta, t) \\
& =\frac{L_{0}}{\pi}+2 \sum_{k=1}^{\infty}\left[a_{2 k}(0) \cos (2 k \theta)+b_{2 k}(0) \sin (2 k \theta)\right]\left(1-4 k^{2}\right) e^{-8 k^{2} t},
\end{aligned}
$$

and thus

$$
\begin{array}{r}
\rho(\theta, t)=\frac{L_{0}}{2 \pi}+\sum_{k=1}^{\infty}\left[a_{2 k-1}(0) \cos ((2 k-1) \theta)+b_{2 k-1}(0) \sin ((2 k-1) \theta)\right]\left(4 k-4 k^{2}\right) \\
+\sum_{k=1}^{\infty}\left[a_{2 k}(0) \cos (2 k \theta)+b_{2 k}(0) \sin (2 k \theta)\right]\left(1-4 k^{2}\right) e^{-8 k^{2} t} .
\end{array}
$$

As we know, $\partial p / \partial t=\eta-w=\partial^{2} w / \partial \theta^{2}=\frac{1}{2}(\partial w / \partial t)$. Integrating this yields

$$
\begin{array}{r}
p(\theta, t)=\frac{L_{0}}{2 \pi}+\sum_{k=1}^{\infty}\left[a_{2 k-1}(0) \cos ((2 k-1) \theta)+b_{2 k-1}(0) \sin ((2 k-1) \theta)\right] \\
+\sum_{k=1}^{\infty}\left[a_{2 k}(0) \cos (2 k \theta)+b_{2 k}(0) \sin (2 k \theta)\right] e^{-8 k^{2} t} .
\end{array}
$$

The formula above is useful because we can use (2-4) and the definition of the support function to draw the graph of the evolving curve $X=(x, y)$ according to the following parametrization of convex curves (see [Green and Osher 1999]):

$$
\begin{aligned}
X & =\langle X, T\rangle T+\langle X, N\rangle N=\frac{\partial p}{\partial \theta} T-p N \\
& =\left(p \sin \theta+\frac{\partial p}{\partial \theta} \cos \theta,-p \cos \theta+\frac{\partial p}{\partial \theta} \sin \theta\right) .
\end{aligned}
$$


At the end of this section, we prove the last part of the Main Theorem.

Lemma 3.5. If the initial curve $X_{0}$ is centrosymmetric, then the flow (1-1) has a global solution on $S^{1} \times[0, \infty)$ and the limiting curve is a circle, and vice versa.

Proof. If the initial curve $X_{0}$ is centrosymmetric and the symmetric center is the origin of the plane, then the support function and the radius of curvature of $X_{0}$ satisfy

$$
p_{0}(\theta)=p_{0}(\theta+\pi), \quad \rho_{0}(\theta)=\rho_{0}(\theta+\pi) .
$$

By the evolution equations of $\rho$ and $\eta$ (see (2-5)), $\partial / \partial t\left(\rho(\theta, t)-\frac{1}{2} \eta(\theta, t)\right)=0$. Thus we get

$$
\rho(\theta, t)-\frac{1}{2} \eta(\theta, t)=\rho_{0}(\theta)-\frac{1}{2} \eta_{0}(\theta)=\rho_{0}(\theta)-\frac{1}{2}\left(\rho_{0}(\theta)+\rho_{0}(\theta+\pi)\right)=0 .
$$

The maximum principle tells us that $0<\frac{1}{2} n \leq \rho(\theta, t) \leq \frac{1}{2} N$ ( $n, N$ are defined in the proof of Lemma 3.1). Since $\frac{1}{2} \eta(\theta, t)$ converges to $L_{0} / 2 \pi, \rho(\theta, t)$ also tends to $L_{0} / 2 \pi$ as $t \rightarrow \infty$. Therefore, the limiting curve is a circle.

If the flow (1-1) has a global solution on $S^{1} \times[0, \infty)$ and the limiting curve is a circle, then (3-14) implies that $a_{2 k-1}(0)=b_{2 k-1}(0)=0$ for $k=1,2, \ldots$ Therefore

$$
p_{0}(\theta)=p_{0}(\theta+\pi)
$$

Namely, $X_{0}$ is centrosymmetric with respect to the origin.

Now, combining Corollary 3.2 and Lemmas 3.3-3.5, we complete the proof of the Main Theorem.

\section{Examples}

In this section, we will illustrate several examples. We have said that the pinching condition (1-2) cannot be omitted in the Main Theorem. In the following, a convex curve is given to show that (3-9) is negative for some $\theta$. Define a function on $S^{1}$ by

$$
p_{0}(\theta)=10-\cos (2 \theta)+\cos (3 \theta)+\frac{1}{8} \cos (5 \theta) \text { for } \theta \in[0,2 \pi] \text {. }
$$

We can construct a closed curve $X_{0}(\theta)=(x(\theta), y(\theta))$ by setting

$$
x=p_{0} \sin \theta+\frac{d p_{0}}{d \theta} \cos \theta, \quad y=-p_{0} \cos \theta+\frac{d p_{0}}{d \theta} \sin \theta .
$$

The support function of $X_{0}(\theta)$ is $p_{0}(\theta)$, and we claim that $X_{0}$ is convex, since we can find that the minimum of the radius of curvature

$$
\rho_{0}(\theta)=\frac{d^{2} p_{0}}{d \theta^{2}}+p_{0}=10+3 \cos (2 \theta)-8 \cos (3 \theta)-3 \cos (5 \theta)
$$




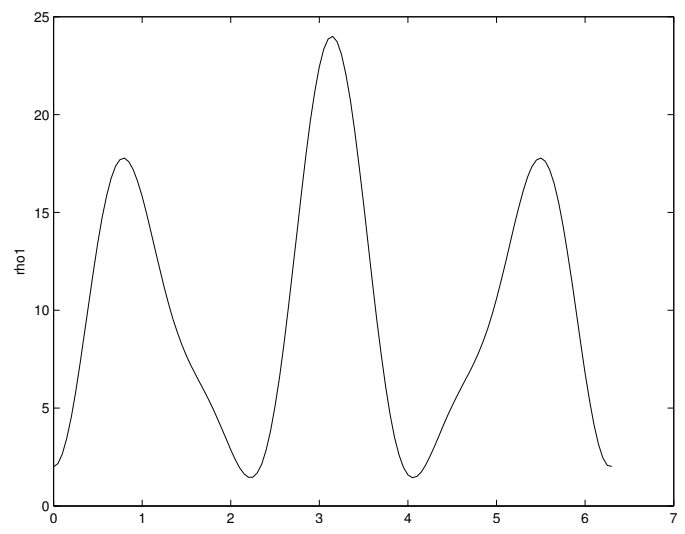

Figure 1. $\rho_{0}(\theta)$.

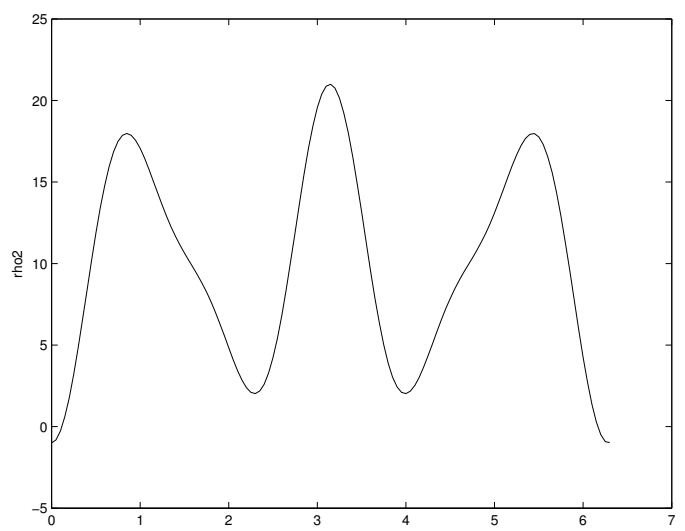

Figure 2. $\rho_{\infty}(\theta)$.

is $1.4409 \ldots$, with the help of Matlab 7.8.0. However, the minimum of

$$
\rho_{\infty}(\theta):=\frac{L_{0}}{2 \pi}+\frac{1}{2}\left(\rho_{0}(\theta)-\rho_{0}(\theta+\pi)\right)=10-8 \cos (3 \theta)-3 \cos (5 \theta)
$$

is -1 . Figures 1 and 2 are the images of functions $\rho_{0}(\theta)$ and $\rho_{\infty}(\theta)$, respectively. A part of the "limiting curve" is given in Figure 3, in which singularities and self-intersections may occur near $x=0$.

If we set the support function of a convex curve $X_{0}$ to be

$$
p_{0}(\theta)=19+2 \cos (2 \theta)+\cos (3 \theta), \quad \text { for } \theta \in[0,2 \pi],
$$

then the radius of curvature is

$$
\rho_{0}(\theta)=19-6 \cos (2 \theta)-8 \cos (3 \theta)
$$




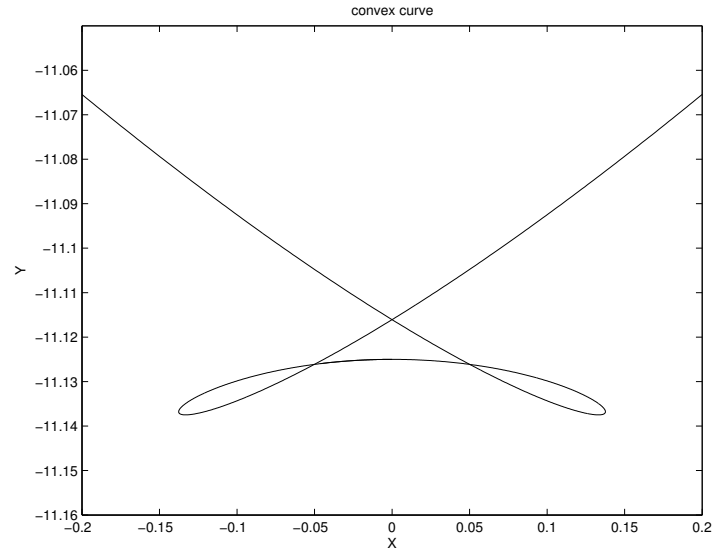

Figure 3. A portion of the limiting curve, with singularities and self-intersection near $x=0$.

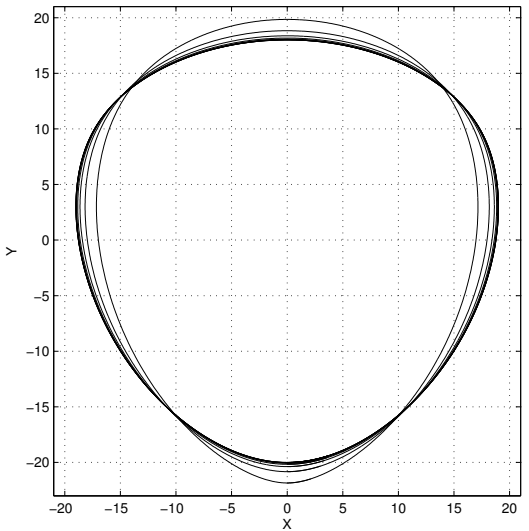

Figure 4. Convergence to a curve of constant width 38 .

with minimum equal to 5 and maximum equal to $30.6366 \ldots$, again using Matlab. Although this convex curve does not satisfy the pinching condition of the Main Theorem, numerical experiment shows that, under the flow (1-1), it keeps its convexity $\left(\rho_{\min }(t) \geq 5\right.$, for every $\left.t \in[0, \infty)\right)$ and converges to a curve of constant width 38 . Figure 4 describes the evolution process.

Our last example is a centrosymmetric convex curve $X_{0}$ with support function

$$
p_{0}(\theta)=15+3 \cos (2 \theta), \quad \text { for } \theta \in[0,2 \pi] .
$$

If $X_{0}$ evolves according to the flow (1-1) then the family of evolving curves converges to a circle. The evolution process is demonstrated in Figure 5. 


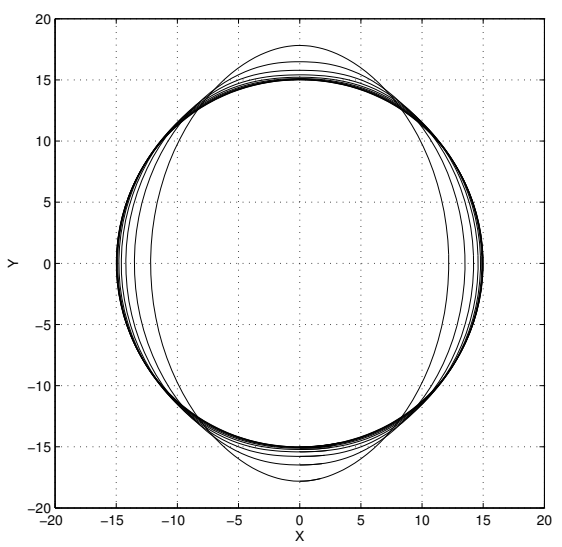

Figure 5. Convergence to a circle.

\section{Acknowledgments}

The authors would like to thank the referee for their careful reading of the manuscript and for giving us some corrections and useful suggestions.

\section{References}

[Andrews 1998] B. Andrews, "Evolving convex curves", Calc. Var. Partial Differential Equations 7:4 (1998), 315-371. MR 99k:58038 Zbl 0931.53030

[Angenent 1991] S. Angenent, "On the formation of singularities in the curve shortening flow", $J$. Differential Geom. 33:3 (1991), 601-633. MR 92c:58016 Zbl 0731.53002

[Cao 2003] F. Cao, Geometric curve evolution and image processing, Lecture Notes in Math. 1805, Springer, Berlin, 2003. MR 2004b:53113 Zbl 01896083

[Chao et al. 2013] X.-L. Chao, X.-R. Ling, and X.-L. Wang, "On a planar area-preserving curvature flow”, Proc. Amer. Math. Soc. 141:5 (2013), 1783-1789. MR 3020863 Zbl 06146523

[Chou and Zhu 2001] K.-S. Chou and X.-P. Zhu, The curve shortening problem, CRC Press, Boca Raton, FL, 2001. MR 2003e:53088 Zbl 1061.53045

[Chow and Tsai 1996] B. Chow and D.-H. Tsai, "Geometric expansion of convex plane curves", J. Differential Geom. 44:2 (1996), 312-330. MR 97m:58041 Zbl 0872.58052

[Gage 1984] M. E. Gage, "Curve shortening makes convex curves circular”, Invent. Math. 76:2 (1984), 357-364. MR 85i:52004 Zbl 0542.53004

[Gage 1986] M. E. Gage, “On an area-preserving evolution equation for plane curves”, pp. 51-62 in Nonlinear problems in geometry (Mobile, AL, 1985), edited by D. M. DeTurck, Contemp. Math. 51, Amer. Math. Soc., Providence, RI, 1986. MR 87g:53003 Zbl 0608.53002

[Gage and Hamilton 1986] M. E. Gage and R. S. Hamilton, "The heat equation shrinking convex plane curves”, J. Differential Geom. 23:1 (1986), 69-96. MR 87m:53003 Zbl 0621.53001

[Grayson 1987] M. A. Grayson, "The heat equation shrinks embedded plane curves to round points", J. Differential Geom. 26:2 (1987), 285-314. MR 89b:53005 Zbl 0667.53001 
[Green and Osher 1999] M. Green and S. Osher, "Steiner polynomials, Wulff flows, and some new isoperimetric inequalities for convex plane curves", Asian J. Math. 3:3 (1999), 659-676. MR 2001j:53089 Zbl 0969.53040

[Groemer 1996] H. Groemer, Geometric applications of Fourier series and spherical harmonics, Encyclopedia Math. Appl. 61, Cambridge University Press, 1996. MR 97j:52001 Zbl 0877.52002

[Gurtin 1993] M. E. Gurtin, Thermomechanics of evolving phase boundaries in the plane, Clarendon, New York, 1993. MR 97k:73001 Zbl 0787.73004

[Hsiung 1981] C. C. Hsiung, A first course in differential geometry, Wiley, New York, 1981. MR 83c:53001 Zbl 0458.53001

[Jiang and Pan 2008] L. Jiang and S. Pan, "On a non-local curve evolution problem in the plane", Comm. Anal. Geom. 16:1 (2008), 1-26. MR 2009e:53083 Zbl 1151.35392

[Lin and Tsai 2009] Y.-C. Lin and D.-H. Tsai, "Evolving a convex closed curve to another one via a length-preserving linear flow", J. Differential Equations 247:9 (2009), 2620-2636. MR 2010j:53135 Zbl 1187.35119

[Ma and Cheng 2014] L. Ma and L. Cheng, "A non-local area preserving curve flow", Geom. Dedicata 171 (2014), 231-247. arXiv 0907.1430

[Ma and Zhu 2012] L. Ma and A. Zhu, "On a length preserving curve flow", Monatsh. Math. 165:1 (2012), 57-78. MR 2886123 Zbl 1235.35175

[Mao et al. 2013] Y. Mao, S. Pan, and Y. Wang, "An area-preserving flow for closed convex plane curves”, Internat. J. Math. 24:4 (2013), Art. ID \#1350029. MR 3062969 Zbl 06180188

[Pan and Yang 2008] S. Pan and J. Yang, "On a non-local perimeter-preserving curve evolution problem for convex plane curves", Manuscripta Math. 127:4 (2008), 469-484. MR 2010h:53099 Zbl 1169.35033

[Pan and Zhang 2010] S. Pan and H. Zhang, "On a curve expanding flow with a non-local term", Comm. Contemp. Math. 12:5 (2010), 815-829. MR 2012c:53102 Zbl 1206.35136

[Schneider 1993] R. Schneider, Convex bodies: the Brunn-Minkowski theory, Encyclopedia Math. Appl. 44, Cambridge University Press, 1993. MR 94d:52007 Zbl 0798.52001

[Urbas 1999] J. Urbas, "Convex curves moving homothetically by negative powers of their curvature", Asian J. Math. 3:3 (1999), 635-658. MR 2001m:53119 Zbl 0970.53039

Received September 1, 2013. Revised December 22, 2013.

\section{LAIYUAN GAO}

DEPARTMENT OF MATHEMATICS

TONGJI UNIVERSITY

ShanghaI, 200092

CHINA

5lygao@mail.tongji.edu.cn

Shengliang Pan

DEPARTMENT OF MATHEMATICS

TONGJI UNIVERSITY

SHANGHAI, 200092

CHINA

slpan@tongji.edu.cn 


\title{
PACIFIC JOURNAL OF MATHEMATICS
}

\author{
msp.org/pjm
}

Founded in 1951 by E. F. Beckenbach (1906-1982) and F. Wolf (1904-1989)

\section{EDITORS}

Don Blasius (Managing Editor)

Department of Mathematics

University of California

Los Angeles, CA 90095-1555

blasius@math.ucla.edu

\author{
Paul Balmer \\ Department of Mathematics \\ University of California \\ Los Angeles, CA 90095-1555 \\ balmer@math.ucla.edu \\ Robert Finn \\ Department of Mathematics \\ Stanford University \\ Stanford, CA 94305-2125 \\ finn@math.stanford.edu \\ Sorin Popa \\ Department of Mathematics \\ University of California \\ Los Angeles, CA 90095-1555 \\ popa@math.ucla.edu
}

\author{
Vyjayanthi Chari \\ Department of Mathematics \\ University of California \\ Riverside, CA 92521-0135 \\ chari@math.ucr.edu \\ Kefeng Liu \\ Department of Mathematics \\ University of California \\ Los Angeles, CA 90095-1555 \\ liu@math.ucla.edu \\ Jie Qing \\ Department of Mathematics \\ University of California \\ Santa Cruz, CA 95064 \\ qing@ cats.ucsc.edu
}

\section{PRODUCTION}

Silvio Levy, Scientific Editor, production@msp.org

\section{SUPPORTING INSTITUTIONS}

ACADEMIA SINICA, TAIPEI

CALIFORNIA INST. OF TECHNOLOGY

INST. DE MATEMÁTICA PURA E APLICADA

KEIO UNIVERSITY

MATH. SCIENCES RESEARCH INSTITUTE

NEW MEXICO STATE UNIV.

OREGON STATE UNIV.

\author{
STANFORD UNIVERSITY \\ UNIV. OF BRITISH COLUMBIA \\ UNIV. OF CALIFORNIA, BERKELEY \\ UNIV. OF CALIFORNIA, DAVIS \\ UNIV. OF CALIFORNIA, LOS ANGELES \\ UNIV. OF CALIFORNIA, RIVERSIDE \\ UNIV. OF CALIFORNIA, SAN DIEGO \\ UNIV. OF CALIF., SANTA BARBARA
}

\author{
Daryl Cooper \\ Department of Mathematics \\ University of California \\ Santa Barbara, CA 93106-3080 \\ cooper@math.ucsb.edu \\ Jiang-Hua Lu \\ Department of Mathematics \\ The University of Hong Kong \\ Pokfulam Rd., Hong Kong \\ jhlu@maths.hku.hk \\ Paul Yang \\ Department of Mathematics \\ Princeton University \\ Princeton NJ 08544-1000 \\ yang@math.princeton.edu
}

These supporting institutions contribute to the cost of publication of this Journal, but they are not owners or publishers and have no responsibility for its contents or policies.

See inside back cover or msp.org/pjm for submission instructions.

The subscription price for 2014 is US $\$ 410 /$ year for the electronic version, and \$535/year for print and electronic.

Subscriptions, requests for back issues and changes of subscribers address should be sent to Pacific Journal of Mathematics, P.O. Box 4163, Berkeley, CA 94704-0163, U.S.A. The Pacific Journal of Mathematics is indexed by Mathematical Reviews, Zentralblatt MATH, PASCAL CNRS Index, Referativnyi Zhurnal, Current Mathematical Publications and Web of Knowledge (Science Citation Index).

The Pacific Journal of Mathematics (ISSN 0030-8730) at the University of California, c/o Department of Mathematics, 798 Evans Hall \#3840, Berkeley, CA 94720-3840, is published twelve times a year. Periodical rate postage paid at Berkeley, CA 94704, and additional mailing offices. POSTMASTER: send address changes to Pacific Journal of Mathematics, P.O. Box 4163, Berkeley, CA 94704-0163.

PJM peer review and production are managed by EditFLOW ${ }^{\circledR}$ from Mathematical Sciences Publishers.

\section{PUBLISHED BY}

\section{mathematical sciences publishers \\ nonprofit scientific publishing}

http://msp.org/

(C) 2014 Mathematical Sciences Publishers 


\section{PACIFIC JOURNAL OF MATHEMATICS}

Volume $272 \quad$ No. $1 \quad$ November 2014

Nonconcordant links with homology cobordant zero-framed surgery $\quad 1$ manifolds

JAE CHOON CHA and MARK POWELL

Certain self-homotopy equivalences on wedge products of Moore spaces

Ho Won CHOI and KeE Young LeE

Modular transformations involving the Mordell integral in Ramanujan's lost notebook

Youn-SEO CHOI

The $D$-topology for diffeological spaces

J. DANiEl Christensen, Gordon SinNAMON and ENXIN Wu

On the Atkin polynomials

AHMAD EL-Guindy and Mourad E. H. ISMAIL

Evolving convex curves to constant-width ones by a perimeter-preserving flow

LAIYUAN GaO and Shengliang PAN

Hilbert series of certain jet schemes of determinantal varieties

SudhiR R. GHORPADE, Boyan Jonov and B. A. SeTHuraman

On a Liu-Yau type inequality for surfaces

Oussama Hijazi, Sebastián Montiel and Simon Raulot

Nonlinear Euler sums

ISTVÁN MEZŐ

Boundary limits for fractional Poisson $a$-extensions of $L^{p}$ boundary functions 227 in a cone

LEI QIAO and TAO ZHAO

Jacobi-Trudi determinants and characters of minimal affinizations

STEVEN V SAM

Normal families of holomorphic mappings into complex projective space concerning shared hyperplanes

LiU Yang, Caiyun Fang and Xuecheng Pang 